\title{
OPEN Predicting women with depressive symptoms postpartum with machine learning methods
}

\author{
Sam Andersson ${ }^{1}$, Deepti R. Bathula ${ }^{2}$, Stavros I. Iliadis ${ }^{1}$, Martin Walter ${ }^{3,4,5}$ \& \\ Alkistis Skalkidou ${ }^{1 凶}$
}

Postpartum depression (PPD) is a detrimental health condition that affects $12 \%$ of new mothers. Despite negative effects on mothers' and children's health, many women do not receive adequate care. Preventive interventions are cost-efficient among high-risk women, but our ability to identify these is poor. We leveraged the power of clinical, demographic, and psychometric data to assess if machine learning methods can make accurate predictions of postpartum depression. Data were obtained from a population-based prospective cohort study in Uppsala, Sweden, collected between 2009 and 2018 (BASIC study, n = 4313). Sub-analyses among women without previous depression were performed. The extremely randomized trees method provided robust performance with highest accuracy and well-balanced sensitivity and specificity (accuracy $73 \%$, sensitivity $72 \%$, specificity $75 \%$, positive predictive value $33 \%$, negative predictive value $94 \%$, area under the curve $81 \%$ ). Among women without earlier mental health issues, the accuracy was $64 \%$. The variables setting women at most risk for PPD were depression and anxiety during pregnancy, as well as variables related to resilience and personality. Future clinical models that could be implemented directly after delivery might consider including these variables in order to identify women at high risk for postpartum depression to facilitate individualized follow-up and cost-effectiveness.

Postpartum depression (PPD), defined as having an episode of minor or major depression during pregnancy or up to one year after giving birth, is a relatively common condition that affects 8-15\% of new mothers in Sweden every year ${ }^{1,2}$. The etiology of PPD is not well understood, but the condition likely arises from a combination of psychological, psychosocial and biological factors ${ }^{3,4}$. The most well documented biological risk factors for PPD are hypothalamic-pituitary-adrenal axis dysregulation, inflammatory processes, genetic vulnerability, and allopregnanolone withdrawal ${ }^{4}$. The strongest psychosocial factors are previous depression, severe life events, some forms of chronic stress and relationship struggles ${ }^{4,5}$. The role of resilience and personality have been lately also gaining attention ${ }^{6,7}$.

PPD is a condition that can have devastating effects on the mothers, as well as their children ${ }^{8,9}$. Mothers may experience persistent doubts about their ability to care for the child, have difficulties bonding with their child, and also have thoughts about hurting the child ${ }^{2}$. Moreover, PPD can affect a child's development by interfering with the mother-infant relationship ${ }^{10,11}$. For instance, children of mothers with PPD have greater cognitive, behavioral and interpersonal problems compared to children of mothers without PPD ${ }^{12,13}$. Despite PPD being a detrimental health condition for many women, numerous affected women fail to receive adequate care ${ }^{14}$. There exist several effective treatments and interventions for $\mathrm{PPD}^{14-16}$, but they are only cost-effective among high-risk women. The idea of prenatal prediction of PPD has existed for several years and early studies using more traditional methods attempted to predict women at risk by prenatal assessment of critical variables ${ }^{17}$. However, to date, there has been no effective way to predict women at risk for the development of depressive symptoms postpartum.

Traditional statistical methods allow researchers to estimate risks by sequentially analysing the associations mainly between two variables, often controlling for the effect of others. Further, machine learning (ML) methods enable researchers to iteratively and simultaneously analyse multiple interacting associations between variables ${ }^{18}$ as well as to devise data-driven predictive models that then can be evaluated by quantifying the performance metrics across all models in order to find the best predictive model. The power of ML allows for the analysis of

${ }^{1}$ Department of Women's and Children's Health, Uppsala University, 75185 Uppsala, Sweden. ${ }^{2}$ Department of Computer Science and Engineering, Indian Institute of Technology Ropar, Rupnagar, Punjab 140001, India. ${ }^{3}$ Department of Psychiatry and Psychotherapy, University Hospital Jena, Jena, Germany. ${ }^{4}$ Department of Psychiatry and Psychotherapy, Eberhardt Karls University, Tübingen, Germany. ${ }^{5}$ Department of Behavioral Neurology, Leibniz Institute for Neurobiology, Magdeburg, Germany. email: Alkistis.skalkidou@kbh.uu.se 
complex non-linear relationships and even the integration and pooling of multiple different data-types from several sources ${ }^{19-21}$. Over the last decade, there has been a steady increase in the use of ML in medicine and its effects can be observed in many fields including oncology ${ }^{22-25}$, cardiology and hematology $y^{26,27}$, critical care $^{28,29}$, and psychiatry $y^{30-35}$. Importantly, PPD represents a unique case in which a moderately high chance to develop a serious psychiatric condition is coupled with a very precise temporal prediction of when such symptoms are to be expected. As such, and considering PPDs substantial societal burden, ML-based risk classification can be applied in an ideal situation with high expected societal benefit. With approximately 120,000 annual births in Sweden and the typical prevalence of PPD at $12 \%$ among women who nearly in their entirety present with a multitude of adaptations after childbirth, close monitoring of the whole population for early depressive sentinels after childbirth seems hardly feasible in reality. In contrast, close follow-up among high risk groups during midwife or nurse-led postpartum assessments may strongly contribute to more tailored and cost-efficient maternal perinatal mental care services.

However, despite promising results in other fields, relatively few studies have been performed using ML in the field of perinatal mental health. An early study in the field could predict PPD with an accuracy of $84 \%$ by use of multilayer perceptrons and assessment of 16 variables $^{36}$. A recent pilot study used ML algorithms applied to data extracted from electronic health records to show that ML models can be utilized to predict PPD and identify critical variables that conform with known risk variables such as race, demographics, threatened abortion, prenatal mental disorder, anxiety, and an earlier episode of major depression ${ }^{34}$. Another study also developed models to predict PPD, which were then integrated into a mobile application platform to be used by pregnant women $^{37}$, while a recently published study compared four PPD prediction models that comprised demographic, social and mental health data ${ }^{38}$. In the latter study, psychological resilience was pointed out as an important predictive factor. However, these studies have been limited by either sample size or richness of data. Finally, in a recently published study, Zhang et al. proposed a machine learning based framework for PPD risk prediction in pregnancy, using electronic health record data ${ }^{39}$.

To date, our study is the first using a population-based, large and rich dataset, including a wide range of clinical and psychometric self-report and medical journal-derived variables and evaluating a range of different ML algorithms against each other, and also after stratification for earlier or pregnancy depression, to provide a robust screening tool, at discharge from the delivery ward, for predicting women at risk for developing depressive symptoms later in the postpartum period.

Hence, we aim to predict women at risk for depressive symptoms at 6 weeks postpartum, from clinical, demographic, and psychometric questionnaire data available after childbirth, by use of machine learning methods.

\section{Results}

Descriptive statistics. Table 1 shows summary statistics of the study population by depressive symptom status at 6 weeks postpartum. Results are presented as frequencies and relative frequencies within EPDS status $[\mathrm{N}(\%)]$ or median (interquartile range) for sociodemographic, clinical and questionnaire variables. Of the 4313 participants in the study, 577 had depressive symptoms at 6 weeks postpartum. The mean age for both groups was 31 years. Differences were seen among women with depressive symptoms and women without depressive symptoms across sociodemographic variables like education, employment, and country of origin, as well as many other variables known as risk factors for postpartum depression. A greater proportion of women with depressive symptoms postpartum did not receive adequate support from their partner and were not breastfeeding.

Classification graphs. To evaluate whether ML can predict women with depressive symptoms, two datasets were used, namely the BP variables and the combined dataset, that includes the BP variables and three psychometric questionnaires (RS, SOC, and VPSQ). Performance of different ML models was first evaluated for the BP data (Fig. 1). The performance metrics for Ridge Regression, LASSO Regression, Gradient Boosting Machines, Distributed Radom Forests (DRF), Extreme Randomized Forests (XRT), Naïve Bayes and Stacked Ensembles models are shown. Balanced accuracy, NPV and AUC were quite similar across the models, with accuracy reaching $72 \%$ and AUC 79\% for XRT. NPV was over $92 \%$ for all models. Sensitivity was quite low and together with specificity and PPV, they varied between the models. Sensitivity was highest for DRF at $84 \%$, while only $65 \%$ for XRT; DRF had though the lowest specificity and PPV. The highest PPV was observed for Ridge Regression and Stacked Ensemble, at $41 \%$.

Performance of different ML models was then evaluated for the combined dataset, even including psychometric measures (Fig. 2). Performance metrics for the same models showed that NPV was still over $90 \%$ for all models, but otherwise, similar levels of accuracy and AUC were observed. More variability among the models was observed for sensitivity, specificity and PPV. XTR had the highest accuracy (at 73\%) and AUC (at $81 \%$ ) among all models, with a balance in sensitivity at $72 \%$ and specificity at $75 \%$; PPV was at $33 \%$ and NPV at $94 \%$. As this balancing act is an essential attribute of predictive models based on imbalanced datasets the subsequent experimental analysis was provided using only XRT.

Comparative performance of the XRT model using all variables, the top 50\%, and the top 25\% variables, for both the BP and the combined dataset is shown in Fig. 3. There was an apparent trade-off between model sensitivity and specificity, which were both affected by dataset used and percent of variables included (Fig. 3). Sensitivity was highest with use of only $25 \%$ of the combined dataset, while specificity was highest with the use of the top $50 \%$ of the BP dataset. None among the other measures were greatly affected by either dataset used or percent of variables included (a trend to lower PPV when $25 \%$ of variables used was noted). The AUC curves corresponding to Figs. 2 and 3 are available in the supplementary material (Supplementary Figure 1). 
EPDS $^{\text {a }}$ at 6 weeks postpartum

Characteristics

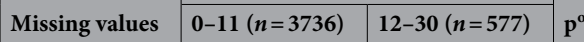

$\mathrm{N}(\%)$ or median $\left(\mathrm{IQR}^{\mathrm{b}}\right)$

Age (years)

BMI $\left(\mathrm{kg} / \mathrm{m}^{2}\right)$ before pregnancy

Height at gestational week $17(\mathrm{~cm})$

University level education (vs. less)

Employment

Full-time work

Part-time work

Studying

Maternity leave

Sick-leave

Unemployed

Marital status (single vs. married/cohabiting)

Country of origin (scandinavia vs. other)

Depression history ${ }^{\mathrm{c}}$

Contact with psychiatrist (self-reported) ${ }^{\mathrm{d}}$

Contact with psychologist (self-reported) ${ }^{\mathrm{d}}$

Hypomanic episodes (1 or more vs. none) ${ }^{\mathrm{d}}$

Premenstrual syndrome history (based on the ICD and ACOG criteria) ${ }^{\mathrm{d}}$

History of pregnancy loss

Parity

\begin{tabular}{|l|l|}
\hline 0 & \\
\hline 1 & \\
\hline 2 or more &
\end{tabular}

Smoking, ever

Snuff, before pregnancy

Sleep before pregnancy (hours per day)

$<6$

$6-8$

$>8$

Migraine $^{\mathrm{d}}$

Irritable bowel syndrome

Alcohol problems $^{\mathrm{d}}$

Allergies $^{\mathrm{d}}$

Endocrine problems ${ }^{\mathrm{d}}$

Hypertension $^{\mathrm{d}}$

Pain problems ${ }^{\mathrm{d}}$

Intimate partner violence ${ }^{\mathrm{d}}$

Pregnancy-related variables

Planned pregnancy

Assisted reproductive technology treatment

Several ultrasounds during gestation

Fear of childbirth

No fear

Fear of caesarean section

Fear of vaginal delivery

Severe fear

Visit at fear of childbirth support unit

Negative delivery expectations

Pregnancy nausea

No

Yes, without medication

Yes, with medication

SSRI $^{f}$ use during pregnancy

Anxiety during pregnancy ${ }^{g}$

Continued

\begin{tabular}{|c|c|c|c|}
\hline 3 & $31(6)$ & $31(6)$ & 0.247 \\
\hline 162 & $22.8(4.4)$ & $23.3(5.5)$ & 0.001 \\
\hline 151 & $168(9)$ & $167(8)$ & 0.305 \\
\hline 268 & $2734(78 \%)$ & $387(72 \%)$ & 0.001 \\
\hline \multirow[t]{7}{*}{264} & & & $<0.001$ \\
\hline & $2291(65 \%)$ & $310(57 \%)$ & \\
\hline & $696(20 \%)$ & $102(19 \%)$ & \\
\hline & $242(7 \%)$ & $49(9 \%)$ & \\
\hline & $110(3 \%)$ & $15(3 \%)$ & \\
\hline & $89(3 \%)$ & $26(5 \%)$ & \\
\hline & $78(2 \%)$ & $41(7 \%)$ & \\
\hline 9 & $55(1.5 \%)$ & $16(2.8 \%)$ & 0.022 \\
\hline 184 & $3311(93 \%)$ & 499 (91\%) & 0.193 \\
\hline 185 & $959(27 \%)$ & $296(54 \%)$ & $<0.001$ \\
\hline 250 & $285(8 \%)$ & $98(18 \%)$ & $<0.001$ \\
\hline 250 & $1190(34 \%)$ & $299(55 \%)$ & $<0.001$ \\
\hline 270 & $141(4.0 \%)$ & $50(9.3 \%)$ & $<0.001$ \\
\hline 303 & $169(4.9 \%)$ & $79(15 \%)$ & $<0.001$ \\
\hline 2072 & $21(1.1 \%)$ & $5(1.6 \%)$ & 0.477 \\
\hline \multirow[t]{4}{*}{303} & & & 0.072 \\
\hline & $1934(55 \%)$ & $309(60 \%)$ & \\
\hline & $1082(31 \%)$ & $154(30 \%)$ & \\
\hline & $477(14 \%)$ & $54(10 \%)$ & \\
\hline 175 & $1157(32 \%)$ & $197(36 \%)$ & 0.096 \\
\hline 402 & $251(7.4 \%)$ & $40(7.6 \%)$ & 0.846 \\
\hline \multirow[t]{4}{*}{179} & & & 0.005 \\
\hline & $124(3 \%)$ & $32(6 \%)$ & \\
\hline & $2927(82 \%)$ & $421(77 \%)$ & \\
\hline & $534(15 \%)$ & $96(17 \%)$ & \\
\hline 295 & $584(17 \%)$ & $120(22 \%)$ & 0.002 \\
\hline 295 & $127(3.7 \%)$ & $32(5.9 \%)$ & 0.011 \\
\hline 295 & $5(0.1 \%)$ & $5(0.9 \%)$ & 0.001 \\
\hline 295 & $764(22 \%)$ & $119(22 \%)$ & 0.951 \\
\hline 295 & $124(3.6 \%)$ & $25(4.6 \%)$ & 0.220 \\
\hline 295 & $55(1.6 \%)$ & $14(2.6 \%)$ & 0.091 \\
\hline 295 & $137(3.9 \%)$ & $43(8.0 \%)$ & $<0.001$ \\
\hline 275 & $362(10 \%)$ & $107(20 \%)$ & $<0.001$ \\
\hline
\end{tabular}

\begin{tabular}{|c|c|c|c|}
\hline 288 & $481(14 \%)$ & $121(22 \%)$ & $<0.001$ \\
\hline 424 & $369(11 \%)$ & $46(8.7 \%)$ & 0.120 \\
\hline 298 & $2241(65 \%)$ & $371(69 \%)$ & 0.048 \\
\hline \multirow[t]{5}{*}{254} & & & $<0.001$ \\
\hline & $2774(79 \%)$ & $336(62 \%)$ & \\
\hline & $339(9.6 \%)$ & $73(14 \%)$ & \\
\hline & $208(5.8 \%)$ & $61(11 \%)$ & \\
\hline & $200(5.6 \%)$ & $68(13 \%)$ & \\
\hline 250 & $77(2.2 \%)$ & $19(3.5 \%)$ & 0.061 \\
\hline 305 & $504(15 \%)$ & $131(25 \%)$ & $<0.001$ \\
\hline \multirow[t]{4}{*}{281} & & & $<0.001$ \\
\hline & $705(20 \%)$ & $74(14 \%)$ & \\
\hline & $2313(66 \%)$ & $363(67 \%)$ & \\
\hline & $476(14 \%)$ & $101(19 \%)$ & \\
\hline 368 & $132(3.9 \%)$ & $39(7.5 \%)$ & 0.001 \\
\hline 237 & $1032(29 \%)$ & $387(72 \%)$ & $<0.001$ \\
\hline
\end{tabular}




\begin{tabular}{|c|c|c|c|c|}
\hline \multirow[b]{2}{*}{ Characteristics } & \multirow[b]{2}{*}{ Missing values } & \multicolumn{2}{|c|}{ EPDS $^{\text {a }}$ at 6 weeks postpartum } & \multirow[b]{2}{*}{$\mathbf{p}^{\mathbf{o}}$} \\
\hline & & $0-11(n=3736)$ & $12-30(n=577)$ & \\
\hline Depression during pregnancy ${ }^{\mathrm{h}}$ & 162 & $472(13 \%)$ & $298(53 \%)$ & $<0.001$ \\
\hline Sleep during pregnancy (hours per day) ${ }^{\mathrm{i}}$ & 266 & & & $<0.001$ \\
\hline$<6$ & & $322(9 \%)$ & $98(18 \%)$ & \\
\hline $6-8$ & & $2340(67 \%)$ & $332(61 \%)$ & \\
\hline$>8$ & & $843(24 \%)$ & $112(21 \%)$ & \\
\hline Self-reported pregnancy complications (any) ${ }^{j}$ & 354 & $1471(42.7 \%)$ & $215(42.0 \%)$ & 0.771 \\
\hline Gestational diabetes $^{j}$ & 136 & $35(1.0 \%)$ & $19(3.5 \%)$ & $<0.001$ \\
\hline Preeclampsia $^{j}$ & 136 & $117(3.2 \%)$ & $23(4.2 \%)$ & 0.242 \\
\hline Anaemia $^{j}$ & 354 & $103(3.0 \%)$ & $21(4.1 \%)$ & 0.177 \\
\hline Hypertension & 347 & $165(4.8 \%)$ & $41(8.0 \%)$ & 0.002 \\
\hline Symphysiolysis $^{j}$ & 683 & $1212(39 \%)$ & $228(47 \%)$ & $<0.001$ \\
\hline Pregnancy length (days) & 194 & $280(13)$ & $280(14)$ & 0.103 \\
\hline \multicolumn{5}{|l|}{ Childbirth-related variables } \\
\hline Delivery month & 1 & $6(6)$ & $6(6)$ & 0.386 \\
\hline Induction & 301 & $651(18.6 \%)$ & $100(19.3 \%)$ & 0.697 \\
\hline Mode of delivery & 0 & & & 0.107 \\
\hline Spontaneous vaginal & & $2795(75 \%)$ & $402(70 \%)$ & \\
\hline Elective caesarean section & & $271(7 \%)$ & $47(8 \%)$ & \\
\hline Acute caesarean section & & $320(9 \%)$ & $64(11 \%)$ & \\
\hline Emergency caesarean section & & $33(1 \%)$ & $7(1 \%)$ & \\
\hline Instrumental delivery & & $317(8 \%)$ & $57(10 \%)$ & \\
\hline Postpartum hemorrhage $(\geq 1000 \mathrm{ml}$ vs. $<1000 \mathrm{ml}$ ) & 301 & $221(6.3 \%)$ & $46(8.9 \%)$ & 0.028 \\
\hline Epidural anaesthesia & 286 & $1306(37 \%)$ & $238(46 \%)$ & $<0.001$ \\
\hline Laceration (Grade III/IV vs. I/II) & 146 & $103(2.8 \%)$ & $14(2.6 \%)$ & 0.712 \\
\hline \multicolumn{5}{|l|}{ Infant-related variables } \\
\hline Gender (female) & 171 & $1733(48 \%)$ & $269(50 \%)$ & 0.458 \\
\hline Birthweight (kg) & 204 & $3.6(0.65)$ & $3.6(0.70)$ & 0.201 \\
\hline Birth length $(\mathrm{cm})$ & 219 & $51(2.5)$ & $51(3.0)$ & 0.242 \\
\hline Head circumference $(\mathrm{cm})$ & 1184 & $35(2.0)$ & $35(2.0)$ & 0.672 \\
\hline Apgar score (1st min) & 222 & & & 0.027 \\
\hline $0-3$ & & $26(1 \%)$ & $6(1 \%)$ & \\
\hline $4-6$ & & $111(3 \%)$ & $28(5 \%)$ & \\
\hline $7-10$ & & $3418(96 \%)$ & $502(94 \%)$ & \\
\hline Umbilical artery base deficit & 991 & $-2.9(4.2)$ & $-2.6(4.6)$ & 0.250 \\
\hline Umbilical artery $\mathrm{pH}$ & 949 & $7.3(0.10)$ & $7.3(0.11)$ & 0.519 \\
\hline Newborn admission to neonatal unit & 286 & $300(8.6 \%)$ & $69(13 \%)$ & $<0.001$ \\
\hline \multicolumn{5}{|l|}{ Postpartum variables } \\
\hline Negative delivery experience & 411 & $217(6.4 \%)$ & $85(17 \%)$ & $<0.001$ \\
\hline Partner helpful with infant & 40 & & & $<0.001$ \\
\hline Yes, a lot & & $2334(63 \%)$ & $300(53 \%)$ & \\
\hline Yes, a little & & $1281(35 \%)$ & $235(41 \%)$ & \\
\hline No & & $87(2 \%)$ & $36(6 \%)$ & \\
\hline Breastfeeding & 11 & & & $<0.001$ \\
\hline Yes, exclusive & & $2918(78 \%)$ & $354(62 \%)$ & \\
\hline Yes, non-exclusive & & $576(16 \%)$ & $146(25 \%)$ & \\
\hline No & & $233(6 \%)$ & $75(13 \%)$ & \\
\hline Stressful life events past 6 months ( $3-30$ vs. $0-2$ ) & 13 & $395(11 \%)$ & $129(22 \%)$ & $<0.001$ \\
\hline \multicolumn{5}{|l|}{ Psychometric scales } \\
\hline \multicolumn{5}{|l|}{ ASQ [pregnancy week 32] ${ }^{\mathrm{k}}$} \\
\hline Distance & 2658 & $2.8(1.1)$ & $3.4(1.4)$ & $<0.001$ \\
\hline Insecure and repudiation connection & 2658 & $2.1(0.86)$ & $2.3(1.0)$ & $<0.001$ \\
\hline Trust & 2658 & $4.8(1.0)$ & $4.3(1.3)$ & $<0.001$ \\
\hline Consents & 2658 & $2.9(1.3)$ & $3.7(1.4)$ & $<0.001$ \\
\hline Relation & 2658 & $3.0(1.0)$ & $3.5(1.1)$ & $<0.001$ \\
\hline SOC (total score) [pregnancy week 32] ${ }^{1}$ & 2095 & $154(28)$ & $131(31)$ & $<0.001$ \\
\hline
\end{tabular}




\begin{tabular}{|c|c|c|c|c|}
\hline \multirow[b]{2}{*}{ Characteristics } & \multirow[b]{2}{*}{ Missing values } & \multicolumn{2}{|c|}{ EPDS $^{\text {a }}$ at 6 weeks postpartum } & \multirow[b]{2}{*}{$\mathbf{p}^{\mathbf{o}}$} \\
\hline & & $0-11(n=3736)$ & $12-30(n=577)$ & \\
\hline LITE (number of events) [12 months postpartum $]^{\mathrm{m}}$ & 1696 & $3.0(3.0)$ & $4.0(4.0)$ & $<0.001$ \\
\hline Resilience Scale-14 [pregnancy week 32] & 1982 & $81(15)$ & $72(24)$ & $<0.001$ \\
\hline $\begin{array}{l}\text { Beck anxiety inventory (moderate/severe vs. minimal/mild) [pregnancy } \\
\text { week 32] }\end{array}$ & 2015 & $299(15 \%)$ & $162(50 \%)$ & $<0.001$ \\
\hline SSP-Neuroticism score [pregnancy week 32] ${ }^{\mathrm{n}}$ & 3230 & $328(66)$ & $387(64)$ & $<0.001$ \\
\hline SSP-Aggressiveness score [pregnancy week 32] & 3236 & $195(26)$ & $203(26)$ & 0.001 \\
\hline SSP-Sensation Seeking score [pregnancy week 32] & 3224 & $94(19)$ & $95(17)$ & 0.258 \\
\hline
\end{tabular}

Table 1. Characteristics of the study participants by depression status at 6 weeks postpartum (EPDS ${ }^{\text {a score }}$ $0-11$ vs. $12-30)(n=4313)$. Bold values indicate $p<0.05$. ${ }^{a}$ Edinburgh Postnatal Depression Scale. ${ }^{b}$ Interquartile range. ${ }^{c}$ Self-reported and/or diagnosed by psychiatrist. ${ }^{\mathrm{d}}$ Pregnancy week 17 , self-reported. ${ }^{e} \mathrm{EPDS} \geq 12$ at

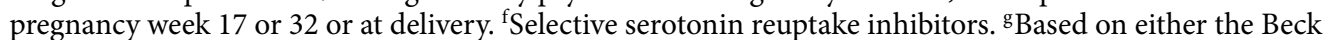
Anxiety Inventory, the State Trait Anxiety Inventory (STAI) or the anxiety subscale of the EPDS (EPDS 3A, items 3-5). ${ }^{\mathrm{h}} \mathrm{EPDS} \geq 12$ at pregnancy week 17,32 or 38 . ${ }^{\text {P Pregnancy week } 32 .}$ 'Self-reported, pregnancy week 32. ${ }^{k}$ Attachment Style Questionnaire. ${ }^{1}$ Sense of Coherence Questionnaire-29. ${ }^{\mathrm{m}}$ Lifetime Instances of Traumatic Events. ${ }^{n}$ Swedish Universities scales of Personality. ${ }^{\circ}$ Based on the Mann-Whitney test for continuous variables and the Pearson Chi-square test for the categorical variables.

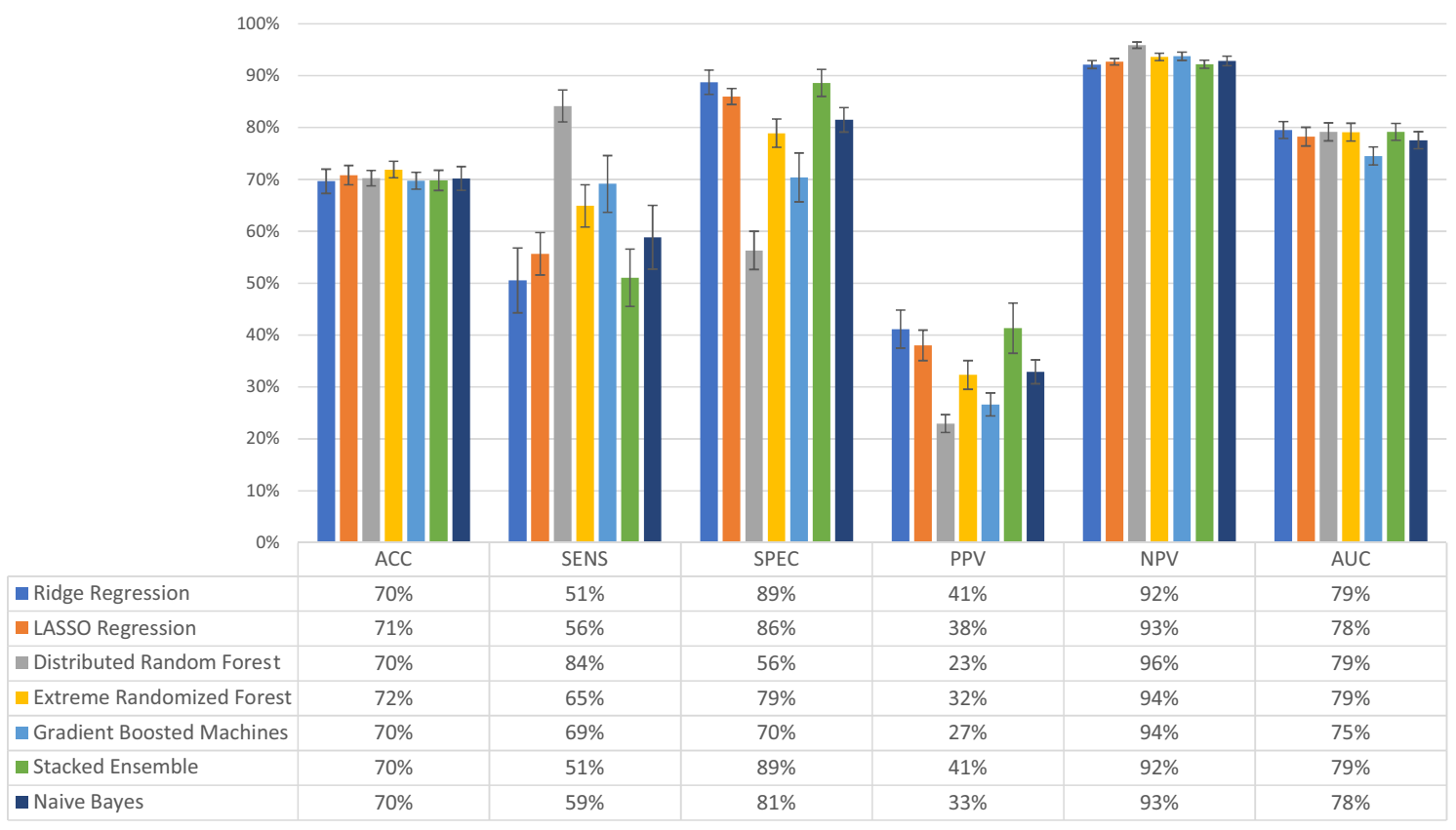

Figure 1. Evaluation of model performance in the dataset containing only background, medical and pregnancy-related variables ( $\mathrm{n}=4277$ women). The models tested were Ridge Regression, LASSO Regression, Distributed Random Forest, Extremely Randomized Trees, Gradient Boosted Machines, Stacked Ensemble, and Naïve Bayes. Models were assessed for accuracy (ACC), sensitivity (SENS), specificity (SPEC), positive predictive value (PPV), negative predictive value (NPV), and area under the curve (AUC), the outcome being depressive symptoms at 6 weeks postpartum. The bars represent the level of performance measures (in percent) and the table below the bar plot presents the exact numerical values. Error bars represent one standard deviation from the mean.

The results for the performance of the XRT models after stratification for previous depression are shown in Fig. 4. For all women, XRT achieved a balanced accuracy of $73 \%$, a sensitivity of $72 \%$, a specificity of $75 \%$, a positive predictive value of $33 \%$, a negative predictive value of $94 \%$ and an AUC of $81 \%$. For women with depression in pregnancy or earlier in life, XRT achieved a balanced accuracy of $69 \%$, a sensitivity of $76 \%$, a specificity of $61 \%$, a positive predictive value of $44 \%$, a negative predictive value of $87 \%$ and an AUC of $77 \%$. For women without any previous depressive episode, balanced accuracy was $64 \%$, sensitivity $52 \%$, specificity $76 \%$, positive predictive value of $13 \%$, negative predictive value $97 \%$ and AUC of $73 \%$ (Fig. 4). Among the results from analyses of the individual questionnaires, no single one achieved an accuracy of more than $70 \%$ (Supplementary Figure 2). 


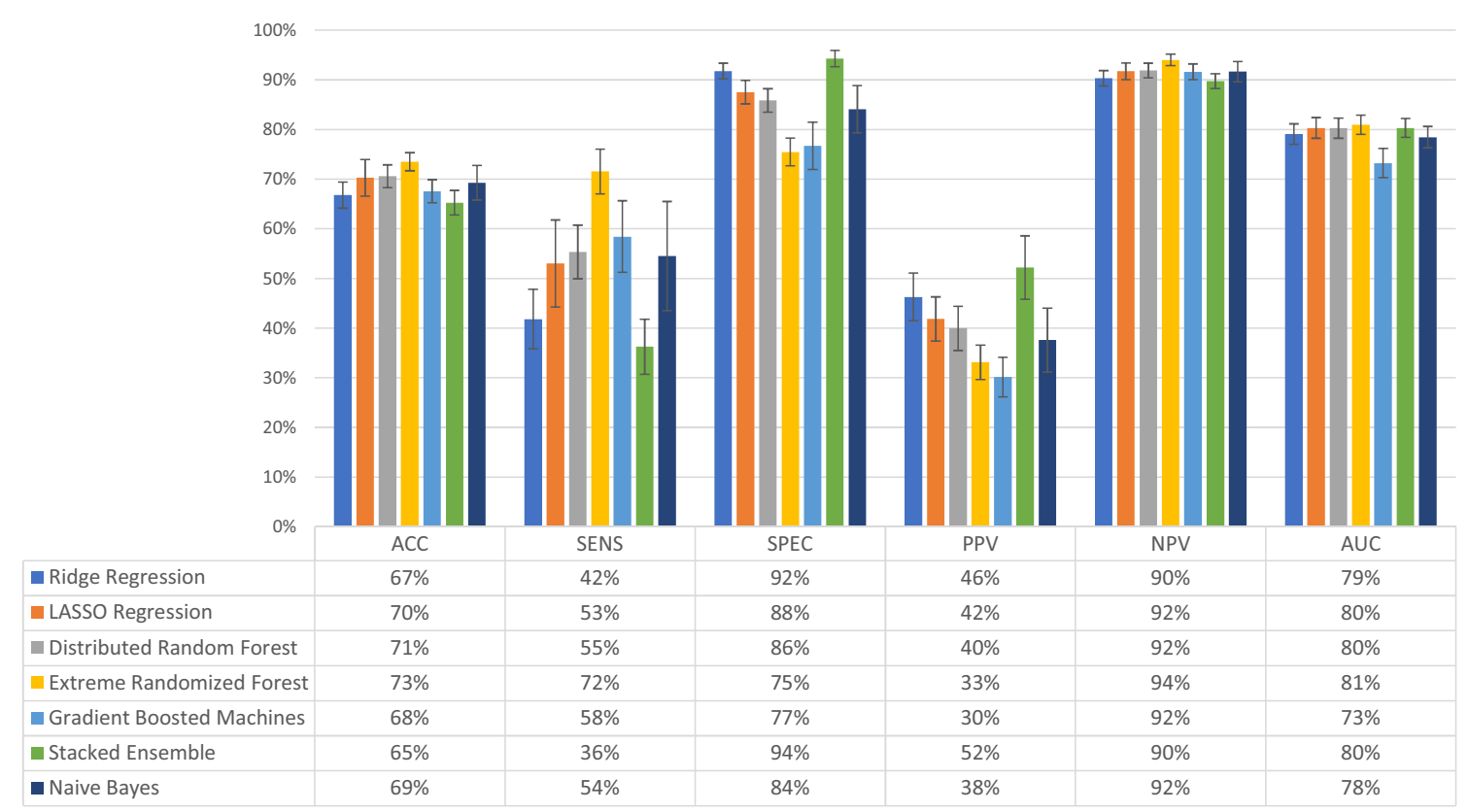

Figure 2. Evaluation of model performance in the total combined dataset ( $\mathrm{n}=2385$ women). The combined dataset contained the background, medical and pregnancy-related variables, as well as answers to the questionnaires Resilience-14, Sense of Coherence-29 and Vulnerable Personality Scale Questionnaire. The models tested were Ridge Regression, LASSO Regression, Distributed Random Forest, Extremely Randomized Trees, Gradient Boosted Machines, Stacked Ensemble, and Naïve Bayes. Models were assessed for accuracy (ACC), sensitivity (SENS), specificity (SPEC), positive predictive value (PPV), negative predictive value (NPV), and area under the curve (AUC), the outcome being depressive symptoms at 6 weeks postpartum. The bars represent the level of performance measures (in percent) and the table below the bar plot presents the exact numerical values. Error bars represent one standard deviation from the mean.

Feature Selection (XRT)

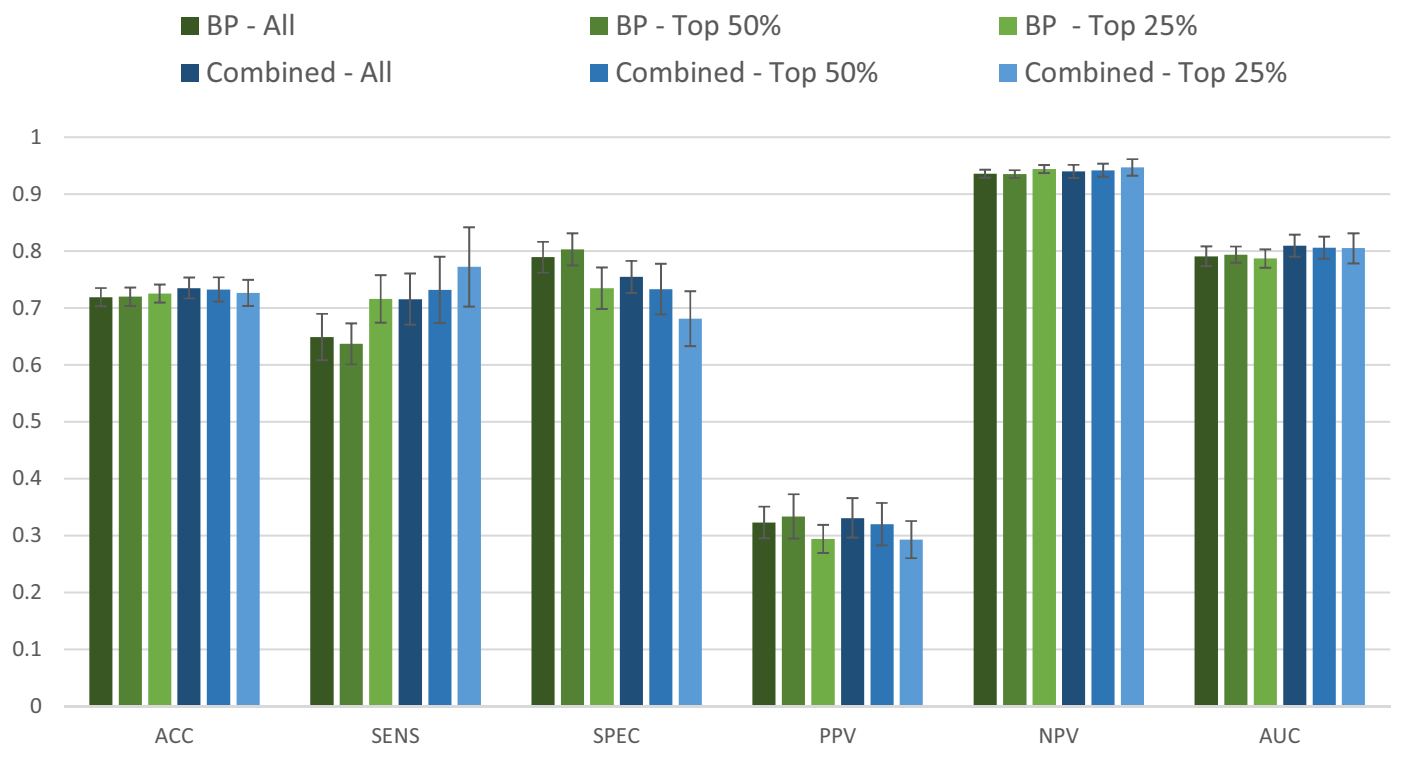

Figure 3. Comparative performance of the dataset containing only background, medical history and pregnancy-related variables (BP) and the combined dataset (BP + RS + SOC + VPSQ). The Extremely Randomized Trees (XRT) algorithm was used to compare the performance of the two datasets for predicting depression at 6 weeks postpartum. Models were assessed for accuracy (ACC), sensitivity (SENS), specificity (SPEC), positive predictive value (PPV), negative predictive value (NPV), and area under the curve (AUC). The variable selection procedure shows results when All (100\%), Top 50\%, and Top 25\% of variables were retained, ranked according to Mean Decrease in Impurity (MDI) relevance. 


\begin{tabular}{|l|r|r|r|}
\hline & All & With Previous Depression & Without Previous Depression \\
\hline \hline ACC & $73.50 \%$ & $69.09 \%$ & $64.63 \%$ \\
\hline SENS & $71.53 \%$ & $51.79 \%$ \\
\hline SPEC & $75.47 \%$ & $76.45 \%$ & $76.47 \%$ \\
\hline PPV & $33.09 \%$ & $61.74 \%$ & $13.06 \%$ \\
\hline NPV & $94.00 \%$ & $44.39 \%$ & $96.64 \%$ \\
\hline AUC & $80.95 \%$ & $86.80 \%$ & $72.54 \%$ \\
\hline
\end{tabular}

Figure 4. Stratified classification graphs for Extreme Randomized Forest (XRT) model, by pregnancy/previous depression status. Results presented for all women (All, $n=2385$, of which $14 \%$ had postpartum depression, PPD), women with depression during current pregnancy or earlier in life (With Previous Depression, $\mathrm{n}=971$, of which $27 \%$ had PPD), and women without any previous depression episode (Without Previous Depression, $\mathrm{n}=1414$, of which $6 \%$ had PPD). For each category, models were assessed for accuracy (ACC), sensitivity (SENS), specificity (SPEC), positive predictive value (PPV), negative predictive value (NPV), and area under the curve (AUC).

Variable importance. The 25 most important variables by MDI based on Distributed Random Forests (DRF) models, considering the women with different previous depression status are shown in Fig. 5. For all women, Anxiety During Pregnancy and Depressive During Pregnancy stand out as the two most important variables (importance level above 0.7) (Fig. 5A). The variables following in importance were questions included in the psychometric instruments, except for history of depression. Similarly, for women with previous depression, Anxiety During Pregnancy and Depressive During Pregnancy stand out as important variables for the presence of depression postpartum (importance level above 0.9) (Fig. 5B). Finally, for women without depression, Anxiety During Pregnancy was the absolutely most important variable (importance level of 1) (Fig. 5C). Even here, variables relating to resilience, sense of coherence and personality followed, but interestingly, variables such as breastfeeding, BMI, traumatic events in childhood, mode of delivery, hypoxia in the newborn and age place among the top 25 variables.

The 25 most important variables based only on BP variables for all women $(n=4313)$ can be found in Fig. 6. The two variables that have an importance level above 0.9 are again Depression During Pregnancy and Anxiety During Pregnancy. The next variable with an importance level above 0.3 is Depression History, while the remaining rate below 0.2 .

Including only the top 20 variables, the AUC is only reduced by $1 \%$ to 0.79 , including just 10 variables reduced the AUC by $2 \%$ to $\sim 0.78$, while after including just 5 variables reduced the AUC by $3 \%$ to $\sim 0.77$. For the previously non-depressed group, including 10 variables gives an AUC of 0.72, and 5 variables an AUC of 0.71 .

\section{Discussion}

In this study, we evaluated a range of different machine learning (ML) methods to predict pregnant women at risk for postpartum depressive (PPD) symptoms. The classification performance of the chosen ML algorithms was not significantly different in regard to accuracy, NPV, AUC measures. However, variations were more pronounced in regard to sensitivity, specificity and PPV. In general, as expected, an inverse relationship is observed in performance with respect to sensitivity and specificity. Furthermore, PPV is considerably lower than NPV due to low prevalence of PPD, as expected.

Overall, XRT provides robust performance with highest accuracy and well-balanced sensitivity and specificity. Addition of resilience and personality self-reported variables to the background, medical history and pregnancy-related variables provides marginal improvement in both accuracy and AUC. It is nevertheless of note that these extra variables boost the sensitivity of the XRT model substantially for only a slight drop in specificity. As this does not depend on the lower sample size used for the second step of analyses involving personality and resilience measures, it could be hypothesized that there is either a certain redundancy between variables, e.g. that low resilience is a core feature among depressed patients during pregnancy, or that anxiety and depression measures, available for all patients, have such a strong predictive value that the further addition of variables does not greatly improve accuracy.

These results suggest a possible benefit of using ML to screen new mothers at discharge from the delivery ward in order to identify those at high risk for postpartum depressive symptoms. However, because of the low PPV across all models, due to the relatively low prevalence of PPD at $12 \%$, one would expect that many women identified at high risk would in the end not get depressed. On the other hand, these methods may nevertheless permit the identification of a high-risk group, to which preventive interventions would be offered in a costeffective way, mainly by avoiding large costs related to full-blown depressive episodes postpartum. These could include the provision of extra support as well as more focused and longitudinal assessments in these mothers. Furthermore, the variables included in the BASIC study refer to easily acquired web-based self-reports, which support their use for screening purposes. Because of the high NPV, we would not expect many women not identified as high risk to develop depression postpartum. As such, the application of our classification algorithms would boost cost-effectiveness, allowing for a tailored resource allocation towards the mothers initially identified at risk versus a more widespread follow up of all mothers; in the low-risk group, assessments could be limited to single timepoints, as is praxis today. As PPD affects more than 16,000 families every year in Sweden alone, with 
A

\section{All Women}

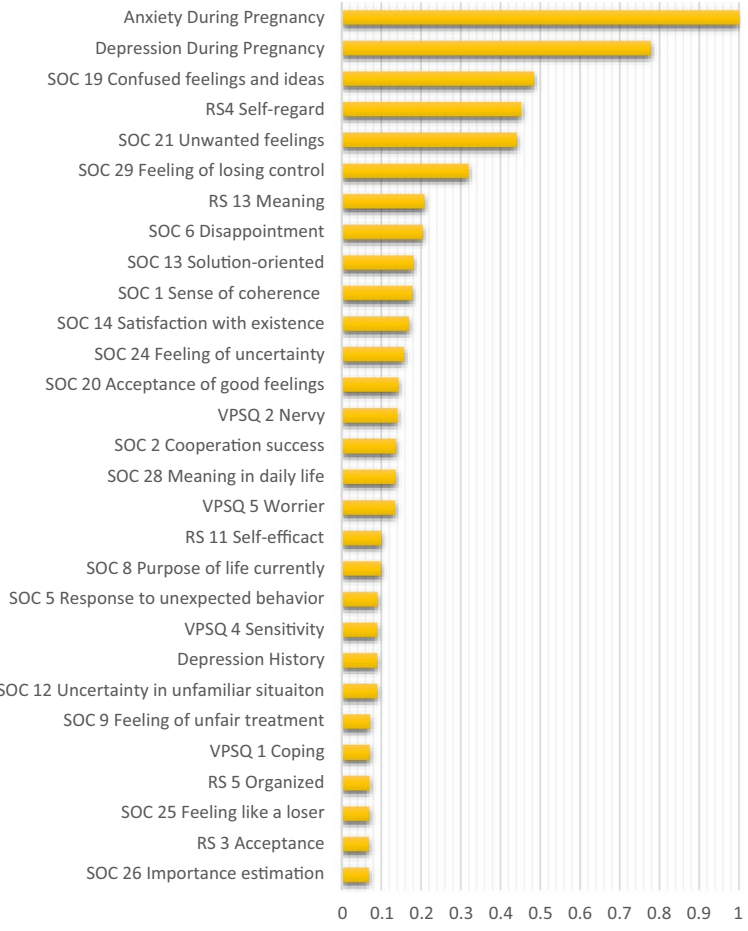

B

\section{With previous/pregnancy depressed}

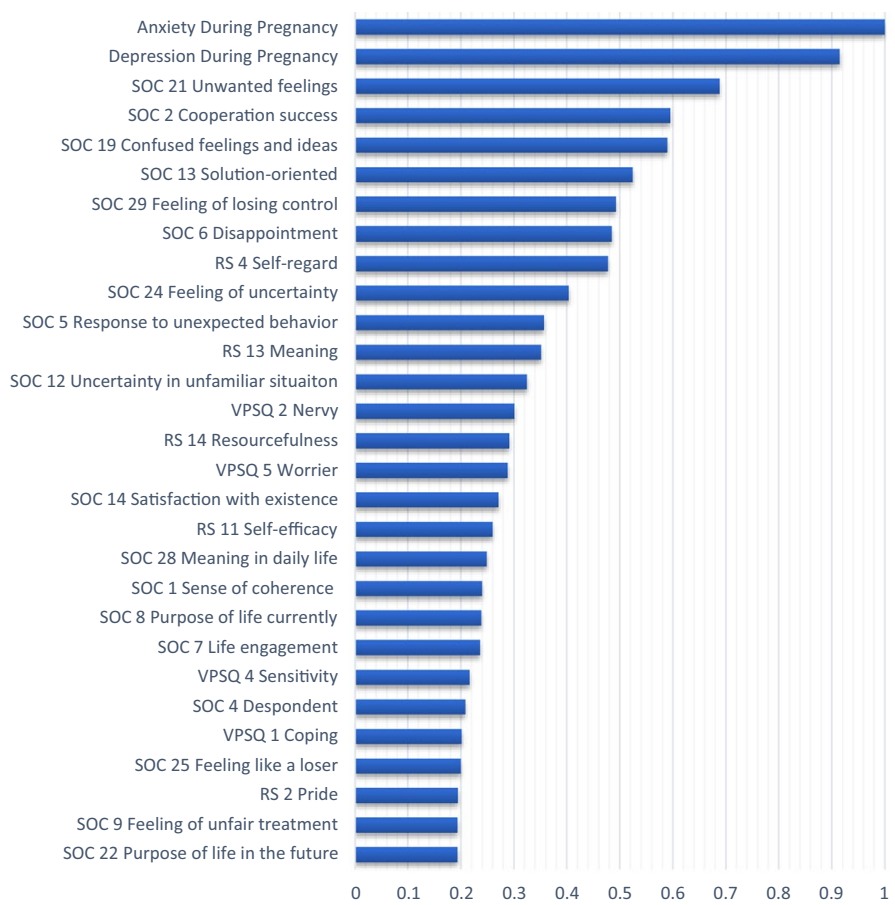

\section{C}

Without previous depression

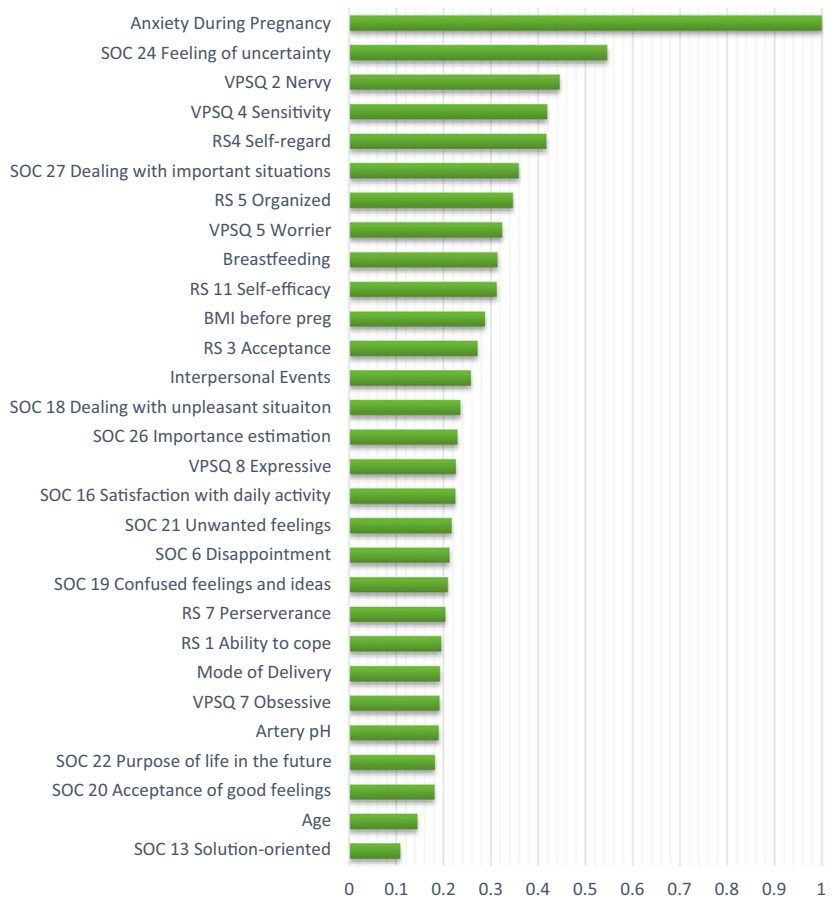

Figure 5. Ranked importance of the assessed variables using the Extremely Randomized Trees (XRT) models in the combined dataset, considering the women with different previous depression status. Results presented for all women (A), All women $(\mathrm{n}=2385),(\mathbf{B})$ women with depression during current pregnancy or earlier in life (Previous/pregnancy depression, $\mathrm{n}=971$ ), and $(\mathbf{C})$ women without any previous depression episode (No previous depression, $\mathrm{n}=1414$ ). The graphs depict the variable importance as a relative measure that is scaled to a maximum of 1.0. The $\mathrm{x}$-axis represents the relative contribution to the classification algorithm of the corresponding feature on the y-axis. 


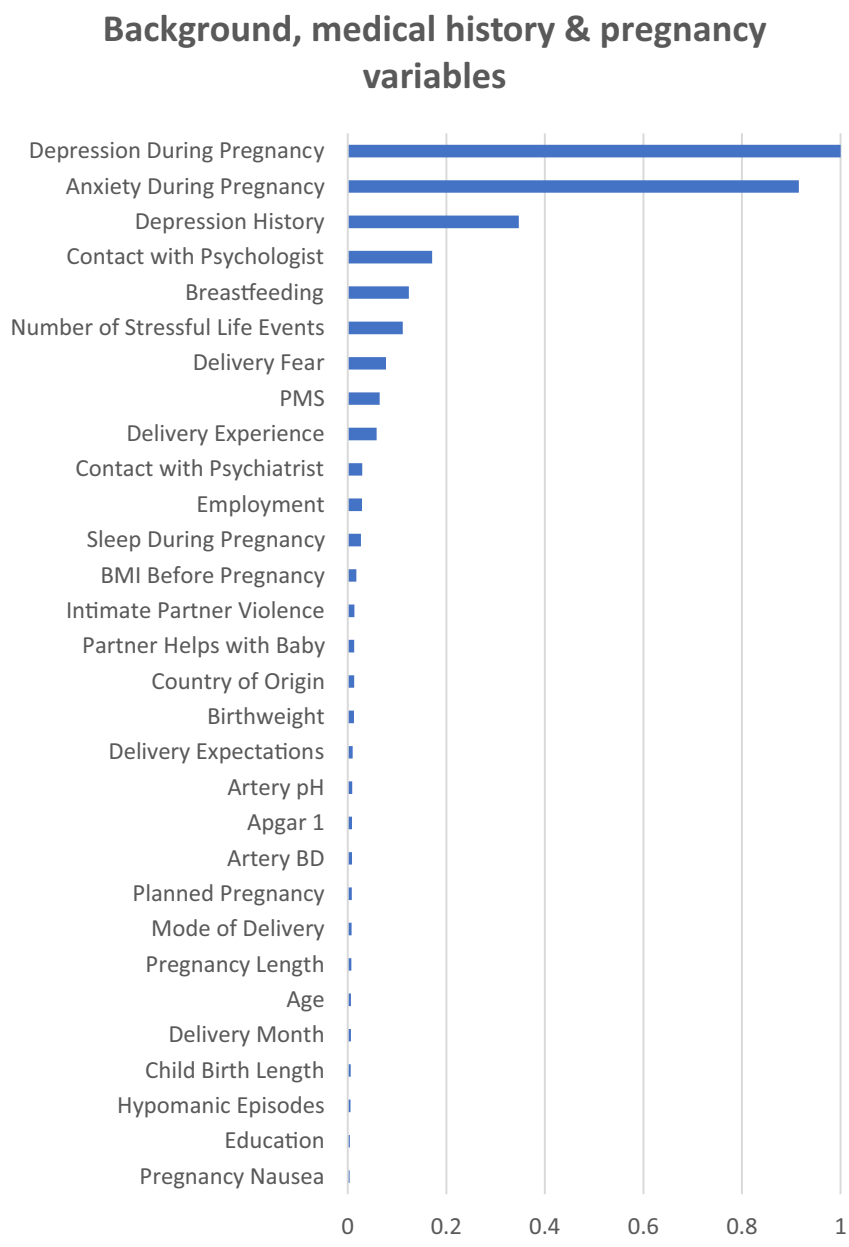

Figure 6. Ranked importance of the assessed background, medical history and pregnancy variables for all women $(n=4277)$ using Extremely Randomized Trees (XRT) models. The top $25 \%$ of the variables are reported. The $\mathrm{x}$-axis represents the relative contribution of the corresponding variable to the classification algorithm.

high associated costs, estimated at $\$ 30,000$ per mother-infant pair for untreated peripartum mood disorders, preventive efforts would have substantial societal benefits ${ }^{40}$.

It is interesting that performance metrics, especially accuracy and AUC, remain stable even when the number of variables used in the models is reduced from 100 to $50 \%$ and even to $25 \%$ of all variables available, and AUC is relatively stable even at $5-10$ variables. As discussed above, this is in line with the thought that there is some redundancy when it comes to the variables included, with depression and anxiety during pregnancy being highly correlated with some background and medical history variables, and possibly mediating their association with PPD. It is thus intriguing to observe that only among non-previously depressed, variables such as breastfeeding, BMI, traumatic interpersonal events in childhood, mode of delivery, infant hypoxia and age are emerging as important for prediction, along with resilience and personality variables, which are otherwise more prominent among those earlier depressed. This is important to have in mind when developing screening strategies; the variables used might need to be adjusted for the group of women with previous depression. Anxiety during pregnancy continues to be very predictive in both groups. The stability of the performance measures however, indicates that an abbreviated survey can be used to screen without significantly affecting predictive power.

Among possible explanations for the somewhat lower accuracy in both the depressed group (earlier or during pregnancy) $(n=971$, accuracy $=69 \%)$ and never-depressed subgroups $(n=1414$, accuracy $=64 \%)$ are the lower sample sizes as well as a relatively decreased variability in the data (the algorithms did not have a big number of examples of alternatives to learn from). Sensitivity is the same in the earlier depressed group, but drops to 52\% in the never depressed group, underlining the difficulty in identifying women at high risk for having their first ever depressive episode after childbirth. In general, the high NPV figure in the never earlier depressed group means that women with a negative screening in that group do not need tighter follow-up; NPV nonetheless drops to $86 \%$ in the earlier depressed group, suggesting that further screening in the postpartum period might still benefit this high-risk group of women.

Our study showed a slightly higher AUC than most earlier studies' best prediction models (79\% by Wang et al. and $78 \%$ by Zhang et al.), though our accuracy of $73 \%$ is lower than the $84 \%$ reported by Tortajada et al. ${ }^{34,36,38}$. However, in the latter study, the main outcome was depression at 32 weeks and not at 6 weeks postpartum, 
genetic data was included and the study sample was more homogeneous since it consisted of SSRI-free Caucasian women. Moreover, a lower EPDS cut-off was used followed by clinical interviews, possibly reducing the risk of misclassification of study cases and controls. Nevertheless, in our study, a clinical evaluation was not possible for practical reasons, due to the much larger study population. Finally, in addition to clinical and environmental variables, information on related gene polymorphisms was also utilized in that study.

Furthermore, Wang et al. identified race, obesity, anxiety, depression, different types of pain, and antidepressant and anti-inflammatory drug use during pregnancy as the most important variables for their prediction models $s^{34}$. These variables differed somewhat from the ones we identified as being most important with the caveat that our model also indicated that anxiety during pregnancy and depression history or depressive symptoms during pregnancy were overwhelmingly the most significant predictors for PPD. It has to be noted that we included many psychometric measures, which followed in importance, e.g. the question 19 on the SOC scale "Do you have very mixed up feelings and ideas?" and question 4 on RS, which measures self-regard ("I am friends with myself"). The population in the BASIC study is quite homogeneous, most participants having a high education, are quite healthy and born in the Nordic countries. Further, the BASIC dataset has no information on race. BMI was also identified in our study as an important variable, both in the BP dataset analysis and the sub-analysis among women without previous depression. Rates of antidepressant use are low. Differences in the analytical approach might also account for some differences in the results.

These findings further illuminate the difficulties in predicting which women will go on to develop postpartum depressive symptoms after childbirth. From the variable importance plots, the most predictive variables for postpartum depressive symptoms, available at the time of discharge from the delivery ward, is to either have anxiety or depressive symptoms during pregnancy. In fact, these two variables are by far the most predictive, along nevertheless with distinct variables related to resilience, sense of coherence and personality. The predictive algorithms reach an accuracy for the whole group of $73 \%$ and AUC of $81 \%$, which is at the limit for possible use in clinical settings. The algorithms might need to be different according to whether women had experience depression before in life. Further studies, possibly using more advanced methods and bigger samples, are warranted.

Very recently, Zhang et al. also proposed a machine learning based framework for PPD risk prediction using electronic health record (EHR) data ${ }^{39}$. While the techniques employed are comparable to our study with similar processing pipeline, they report higher AUC. This increment can be attributed majorly to the substantially larger cohort used in their study. Several ML studies have demonstrated that large datasets lead to lower estimation variance and hence provide better predictive performance. Furthermore, the top predictors also differ between our study due to differences in data sources. Additionally, a PPV higher than that reported in our study would significantly increase the clinical utility of our proposed framework. However, PPV is directly related to the prevalence of PPD in the population studied, which is only about $12 \%$. While the classification threshold of the model can be adjusted to improve PPV, it does not ensure the expected benefit as other evaluation metrics, like sensitivity, specificity and NPV, would be adversely affected. Even Zhang et al. that reported higher AUC values, only report a PPV of $\sim 27 \%$ for the validation site with prevalence of $6.5 \%$, highlighting the issue ${ }^{39}$.

The lack of effective ways that would allow for early prediction of women at risk for depressive symptoms in the postpartum period has been addressed in the Introduction. In fact, the Edinburgh Postnatal Depression Scale is nowadays used as a screening tool for current depression ${ }^{41}$. National guidelines in several countries recommend screening for PPD at 6 to 8 weeks postpartum; however, the suggested target groups of women to be screened vary between countries ${ }^{42-44}$. Also, the use of the EPDS at this time is used to screen for concurrent depression. In contrast, the role of EPDS in pregnancy, in combination with other variables, for early identification of women at risk for development of depressive symptoms later in the postpartum period has not been studied. In our study we do show that high EPDS scores in pregnancy are highly predictive of postpartum depression.

This study had numerous strengths. First, it addresses a novel field, as there are very few studies in the area, none from the Nordic countries, and none of earlier algorithms is being widely used in clinical practice. The large sample size allowed us to train a robust range of different ML algorithms. The richness of the BASIC dataset provided us with the opportunity to investigate the predictive power of a large number of background, medical history, pregnancy and delivery related variables, as well as psychometric questionnaires; the last ones both as total scores but also at individual item level. A key novelty feature of the study in the inclusion of many resilience and personality-related variables, that have been identified in the literature but not included in previous models. We also explore the importance of variables in terms of their predictive power of PPD, an effort directed towards to designing a compact survey to screen for PPD. Finally, the analysis of clinically relevant sub-groups such as women with previous depression or depression during pregnancy gave clinically useful insights.

Some limitations of the study include the non-representative sample in that women born in Scandinavia, with a high education and cohabitating with the child's father were over-represented in the cohort, which makes the findings difficult to generalize to the background population. Sources of selection bias are the exclusion of non-Swedish speaking women as the questionnaires were only offered in the Swedish language, and the fact that more healthy women are more prone to participate in studies of this kind. Not all women self-reported on all variables, but we addressed this problem of missing values with exclusions and imputations where appropriate. Class imbalance in the outcome made the training stages of the algorithms challenging but were also addressed appropriately. Lastly, theoretically, some items from the scales on personality (SSP), and attachment (ASQ) might have had a more prominent role in prediction if they would have been available for a larger proportion of the women in this study. The study by Zhang et al., published after our study was conducted, reported higher AUC and included some predictors lacking in our study ${ }^{39}$. Future studies should make sure to include these important predictive variables for further evaluation.

Depressive symptoms and anxiety during pregnancy are highly predictive factors for women who go on and develop postpartum depressive symptoms, while variables relating to resilience, sense of coherence and 
personality also play a modest role. The predictive algorithms have relatively good accuracy and AUC, with XRT performing best.

\section{Methods}

Data sources. Data for the development of the prediction models were obtained from the "Biology, Affect, Stress, Imaging and Cognition during Pregnancy and the Puerperium" (BASIC) study. BASIC is a populationbased prospective cohort study at the Department of Obstetrics and Gynaecology at Uppsala University Hospital, Uppsala, Sweden ${ }^{7}$. Between September 2009 and November 2018 all pregnant women who were 18 years of age or older, did not have their identities concealed, had sufficient ability to read and understand Swedish and did not have known bloodborne infections and/or non-viable pregnancy as diagnosed by routine ultrasound were invited to participate in the study ${ }^{45}$. Data acquisition in the BASIC study was mainly based on online surveys and questionnaires that the women were asked to fill out during pregnancy at the 17 th and 32 nd gestational week and at 6 weeks, 6 months and 12 months postpartum. The surveys included questions about background characteristics, such as sociodemographic variables, psychological measures, medical information, information on reproductive history, lifestyle and sleep. All questionnaires were self-reported and web-based. Data are also retrieved from the medical journals. The participation rate for the study was $20 \%$ but the cohort had a relatively low attrition rate, with $71 \%$ of the participants remaining in the study at 12 months follow-up ${ }^{45}$.

This study focuses on two subsets of variables from the BASIC study: and (i) background, medical history and pregnancy/delivery variables (BP) and (ii) further psychometric questionnaires (information on exact assessment methods and coding is provided in Table 1 for the background variables and Supplementary Table 1 for the exact questions in the different questionnaires). The BP variables consisted of sociodemographic and lifestyle information, self-reported health, medical history and variables relating to pregnancy and childbirth. This dataset included even information on depression and anxiety symptoms during pregnancy. Depression symptoms were assessed by a score of 12 or more on the Edinburg Postnatal Depression Scale (EPDS) in pregnancy weeks 17, 32 or 38, while anxiety during pregnancy was defined as ratings in the highest quartile on either the State Trait Anxiety Inventory (STAI) ${ }^{46}$, the Beck Anxiety Inventory or the anxiety subscale of the EPDS (EPDS-3A). These variables were available for the majority of the BASIC participants. The total number of interpersonal and noninterpersonal events in the Lifetime Instances of Traumatic Events Scale (LITE) ${ }^{47}$ was also included among BP variables. The BP variables consisted of continuous, discrete, nominal and ordinal categorical variables, measured at various time points during the study.

The extra psychometric scales used were the Attachment Style Questionnaire (ASQ) ${ }^{48}$, the Resilience-14 scale $(\mathrm{RS})^{49,50}$, the Sense of Coherence Scale-29 (SOC) ${ }^{51}$, the Vulnerable Personality Style Questionnaire (VPSQ) ${ }^{52,53}$, and the Swedish Scale of Personalities (SSP) ${ }^{54}$. ASQ, RS, SOC, VPSQ, and SSP were filled out at gestational week 17 or 32, VPSQ and LITE assessments were conducted at 12 months postpartum. All variables were assessed on a Likert scale and coded as ordinal variables. These scales were used for only specific period of time during the course of the BASIC project, different for each scale, and are thus available for different number of women $(\text { Table } 1)^{45}$.

Additionally, the participants of BASIC study were also asked to fill out the EPDS at different time-points during and after pregnancy. The outcome in this study was EPDS score at 6 weeks postpartum, assessing the degree of self-reported depressive symptoms in the early postpartum period. The discrete scores for this timepoint were then aggregated and a cut-off of a score of 12 or higher was used to indicate women with depressive symptoms, in accordance to validation studies for the Swedish population ${ }^{55}$. The number of women in the BASIC study who had completed the EPDS at 6 weeks postpartum and were thus included was 4313.

Ethics declarations. The study has been approved by the Research Ethics Board in Uppsala (Dnr 2009/171, with amendments). All participating women gave written informed consent before being included in the study. All methods were carried out in accordance with relevant guidelines and regulations.

Data pre-processing. The pre-processing consisted of splitting the original BASIC dataset into different subsets. Two subsets were retained for our study, i.e. background \& pregnancy (BP) data and psychometric questionnaire data. Data for twins and women with multiple pregnancies were removed from the dataset, as these are relatively rare, are followed very closely during and after childbirth, and are associated with higher risk for $\mathrm{PPD}^{56,57}$. Explorative data analyses were conducted on individual variables to check their distributions and to identify and remove outliers that were assessed to be non-informative. Psychometric questionnaires and $\mathrm{BP}$ variables that contained information about the women after the time point of the outcome, namely 6 weeks postpartum, were also excluded to avoid inadvertent biases of the results.

SSP was omitted from the analysis due to large number of missing observations, as this survey was used only for few years during recruitment for the BASIC study ${ }^{45}$. Its inclusion would have resulted in a much smaller sample size for the final analysis.

The dataset consists of continuous, nominal and ordinal variables. As continuous variables in the dataset have varying scales, normalization is performed to transform all the variables to a common range from 0 to 1. Furthermore, nominal and ordinal variables that represent non-numerical values are encoded using binary numerical representations for improving the performance of the ML algorithms.

Data imputation. As missing values can drastically impact the performance of ML models, a conservative approach was adopted to handle them. Firstly, samples (rows, corresponding to one pregnancy) with more than $50 \%$ missing values in the included variables were eliminated, and the final number of pregnancies in the ML analyses was 4277 . Next, variables (columns, corresponding to a distinct variable) with more than $25 \%$ missing 
data were also eliminated. Finally, the remaining missing values were imputed from the available data. While continuous variables were imputed using multivariate imputation by chained equations $(\mathrm{MICE})^{58}$, categorical and ordinal variables were imputed with K nearest neighbors' imputation ${ }^{59}$.

\section{Modeling}

Classification techniques. With ML algorithms, there is no one-size-fits-all solution, making it imperative to try multiple alternatives. Consequently, this study explored different ML algorithms for supervised classification that modeled data in different ways. In order to present a comprehensive comparison, the following algorithms were implemented: Ridge Regression, LASSO Regression, Gradient Boosting Machines, Distributed Radom Forests, Extreme Randomized Forest, Naïve Bayes, and Stacked Ensembles. Ridge Regression specializes in analysing multiple regression data with multicollinearity, while LASSO Regression is a type of linear regression that shrinks data values towards a central point, and results in simple, sparse models (i.e. models with fewer parameters). Gradient Boosting Machines (GBM) and Random Forests are ensemble learners. In Distributed Radom Forests (DRF), a subset of features is used to determine the most discriminative thresholds to split the trees on. However, unlike DRF, where one builds an ensemble of deep independent trees, in GBM, we specify an ensemble of weak, shallow successive trees, where each tree is learning and improving on the previous tree. In Extremely Randomized Trees (XRT), instead of using the most discriminative thresholds for the splits, thresholds are drawn at random for each feature and the best of these random thresholds are used as the splitting rule, resulting in lower variance but more bias. XRT are similar to DRF with the caveat of more randomness. Naïve Bayes (NB) is a probabilistic classifier based on Bayes' Theorem. The NB works under the assumption that the presence of any particular feature for a certain outcome is unrelated to the presence of any other feature for that outcome. Thus, despite if the features depend on each other or upon the existence of other features, the NB assumes that all of the features independently contribute to the outcome probability. Stacked Ensemble learns a new model by combining predictions of existing models. Stacked Ensembles are a class of supervised learning algorithms that work by training a meta-learner to find the optimal combination of base learners. Unlike bagging and boosting were the goal is to stack a number of weak learners together, the goal is to stack a number of diverse and strong learners together to optimize learning ${ }^{60}$.

For all the classification algorithms, the outcome measure was the participants' EPDS score at 6 weeks postpartum represented as a binary variable with 12 as cut-off, while predictor variables included the BP variables and psychometric data described above.

Class imbalance. The BASIC dataset, as a population-based sample and in accordance to clinical situations, is predominantly composed of data from women who did not experience PPD at 6 weeks postpartum (less than $10 \%$ of the women representing PPD cases), consequently leading to extreme data class imbalance. ML classifiers trained on such imbalanced datasets usually generate biased results. To mitigate this imbalance, the minority class consisting of women with PPD was oversampled during ML training. Unlike under sampling of majority class consisting of women without PPD, this approach avoids loss of information and leverages all the samples from both classes.

Evaluation metrics. The performance of model prediction of the ML classification algorithms was evaluated using a variety of performance metrics. The performance of each classification model was captured by the Confusion Matrix that formed the basis for other metrics. In addition to the most commonly used classification accuracy, sensitivity (true positive rate) and specificity (false positive rate) are also reported. The positive predictive value (PPV) and negative predictive value (NPV) are also reported. Additionally, a Receiver Operating Characteristic (ROC) curve was specified for each classification to show the relation between the true positive rate and false positive rate. The performance of the classifiers was then summarized by the total area under the ROC curve (AUC), with the higher the AUC (between 0 and 1) indicating a better performance of the classification.

Variable (feature) importance/selection. The success of a ML algorithm does not only depend on good predictive performance but also on generalizability and easy interpretability. Identifying variables that have significant impact on the outcome is valuable, especially in the medical domain. Variable importance using Random Forests models can be calculated using Gini Importance or Mean Decrease in Impurity (MDI $)^{61}$. The MDI relevance of a variable is obtained by calculating how effective the variable is at reducing the uncertainty when creating decision trees. The variable that is most effective and used the most will be ranked as most important.

Analytic strategy. The analytical strategy consisted of breaking the analysis down into steps and iteratively building towards a final classification model, all the while being cognizant of any potential biases introduced by the approach. The workflow is presented in Fig. 7. First, the raw data was split into the BP and the different psychometric questionnaires datasets in order to build predictive models independently on each psychometric questionnaire and to identify the ones with the highest accuracy for classification of PPD. Second, the psychometric questionnaires that yielded the highest accuracies were combined with the BP dataset. Predictions were then performed with the aggregate data (combined dataset). Additional models were trained with reduced datasets resulting from variable selection. Top 50\% and top 25\% variables with MDI were used to train separate classification models to determine the relative contribution of those variables to the prediction. Additionally, stratified analyses were performed, where participants were stratified by a previous history of depression (defined as earlier depression, earlier contact with psychiatrist/psychologist, or depression during pregnancy). 


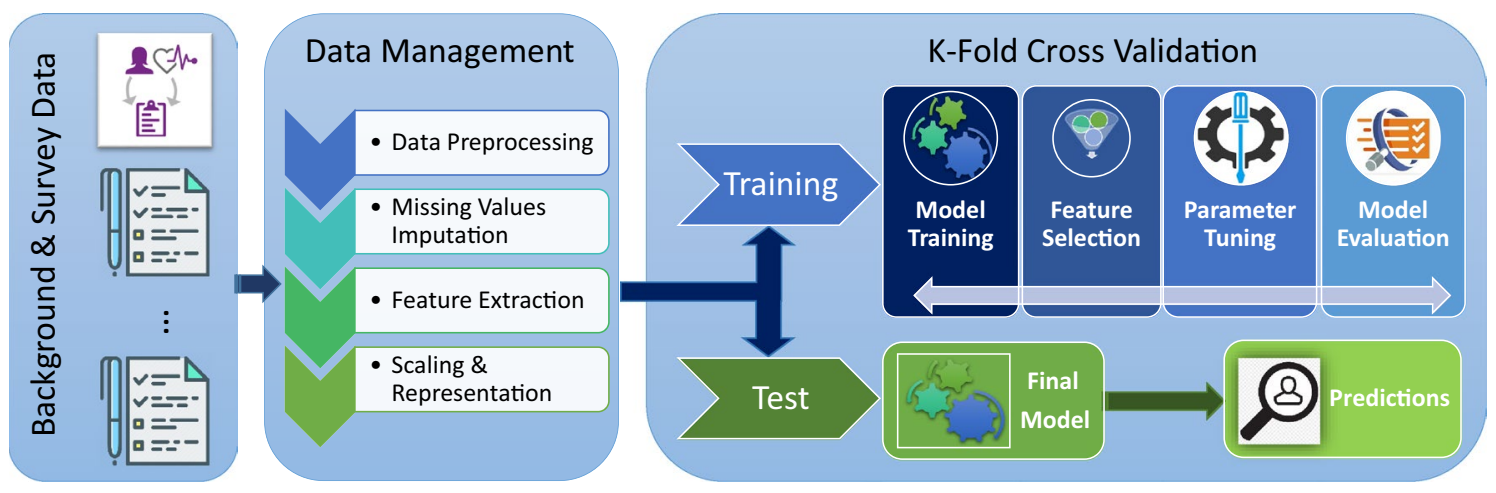

Figure 7. Study workflow and analytical strategy. Data were obtained from the "Biology, Affect, Stress, Imaging and Cognition during Pregnancy and the Puerperium" (BASIC) study, a population-based prospective cohort study in Uppsala, Sweden. Data included in our study comprised (i) background, medical history and pregnancy-related variables (BP) from women, and (ii) further psychometric questionnaires, available at discharge from the delivery ward. The data were processed and either were used to test models or train the machine learning algorithms, to predict depressive symptoms at 6 weeks postpartum.

Based on preliminary analyses, SSP and ASQ did not provide any information gain relative to BP data. Hence, only RS, SOC and VPSQ variables that provided predictive performances comparable to BP variables were included in the aggregate analysis.

\section{Data availability}

The datasets generated and/or analysed during the current study are available from the corresponding author on reasonable request and after data transfer agreements are in place, according to current regulations.

Received: 16 September 2020; Accepted: 15 March 2021

Published online: 12 April 2021

\section{References}

1. American Psychiatric Association. Diagnostic and Statistical Manual of Mental Disorders: DSM-5 5th edn. (American Psychiatric Association, 2013).

2. Fitelson, E., Kim, S., Baker, A. S. \& Leight, K. Treatment of postpartum depression: clinical, psychological and pharmacological options. Int. J. Womens Health 3, 1-14. https://doi.org/10.2147/IJWH.S6938 (2010).

3. Patel, M. et al. Postpartum depression: a review. J. Health Care Poor Underserved 23, 534-542. https://doi.org/10.1353/hpu.2012. 0037 (2012).

4. Yim, I. S., Tanner Stapleton, L. R., Guardino, C. M., Hahn-Holbrook, J. \& Dunkel Schetter, C. Biological and psychosocial predictors of postpartum depression: systematic review and call for integration. Annu. Rev. Clin. Psychol. 11, 99-137. https://doi.org/10. 1146/annurev-clinpsy-101414-020426 (2015).

5. Bloch, M., Daly, R. C. \& Rubinow, D. R. Endocrine factors in the etiology of postpartum depression. Compr. Psychiatry 44, 234-246. https://doi.org/10.1016/S0010-440X(03)00034-8 (2003).

6. Asif, S. et al. Severe obstetric lacerations associated with postpartum depression among women with low resilience-a Swedish birth cohort study. BJOG https://doi.org/10.1111/1471-0528.16271 (2020).

7. Iliadis, S. I. et al. Personality and risk for postpartum depressive symptoms. Arch. Womens Ment. Health 18, 539-546. https://doi. org/10.1007/s00737-014-0478-8 (2015).

8. Committee on Obstetric Practice. American College of Obstetricians and Gynecologists Committee opinion no. 630: screening for perinatal depression. Obstet. Gynecol. 125, 1268-1271 (2015).

9. Ko, J. Y., Rockhill, K. M., Tong, V. T., Morrow, B. \& Farr, S. L. Trends in postpartum depressive symptoms-27 States, 2004, 2008, and 2012. MMWR Morb. Mortal Wkly. Rep. 66, 153-158. https://doi.org/10.15585/mmwr.mm6606al (2017).

10. Dennis, C. L. \& McQueen, K. The relationship between infant-feeding outcomes and postpartum depression: a qualitative systematic review. Pediatrics 123, e736-e751. https://doi.org/10.1542/peds.2008-1629 (2009).

11. Slomian, J., Honvo, G., Emonts, P., Reginster, J.-Y. \& Bruyère, O. Consequences of maternal postpartum depression: a systematic review of maternal and infant outcomes. Womens Health 15, 174550651984404. https://doi.org/10.1177/1745506519844044 (2019).

12. Moore Simas, T. A. et al. Matched cohort study of healthcare resource utilization and costs in young children of mothers with postpartum depression in the United States. J. Med. Econ. 23, 174-183. https://doi.org/10.1080/13696998.2019.1679157 (2020).

13. Murray, L., Woolgar, M., Cooper, P. \& Hipwell, A. Cognitive vulnerability to depression in 5-year-old children of depressed mothers. J. Child Psychol. Psychiatry 42, 891-899. https://doi.org/10.1111/1469-7610.00785 (2001).

14. Nguyen, J. A literature review of alternative therapies for postpartum depression. Nurs. Womens Health 21, 348-359. https://doi. org/10.1016/j.nwh.2017.07.003 (2017).

15. The Management of Depression During Pregnancy. A report from the American Psychiatric Association and the American College of Obstetricians and Gynecologists. Obstet. Gynecol. 114, 703-713. https://doi.org/10.1097/AOG.0b013e3181ba0632 (2009).

16. Dennis, C.-L. \& Hodnett, E. D. Psychosocial and psychological interventions for treating postpartum depression. Cochrane Database Syst. Rev. https://doi.org/10.1002/14651858.CD006116.pub2 (2007).

17. Righetti-Veltema, M., Conne-Perréard, E., Bousquet, A. \& Manzano, J. Risk factors and predictive signs of postpartum depression. J. Affect. Disord. 49, 167-180. https://doi.org/10.1016/s0165-0327(97)00110-9 (1998).

18. Lee, Y. et al. Applications of machine learning algorithms to predict therapeutic outcomes in depression: a meta-analysis and systematic review. J. Affect. Disord. 241, 519-532. https://doi.org/10.1016/j.jad.2018.08.073 (2018). 
19. Bzdok, D. \& Meyer-Lindenberg, A. Machine learning for precision psychiatry: opportunities and challenges. Biol. Psychiatry Cognit. Neurosci. Neuroimaging 3, 223-230. https://doi.org/10.1016/j.bpsc.2017.11.007 (2018).

20. Friston, K. J., Redish, A. D. \& Gordon, J. A. Computational nosology and precision psychiatry. Comput. Psychiatry 1, 2-23. https:// doi.org/10.1162/CPSY_a_00001 (2017).

21. Rohart, F., Gautier, B., Singh, A. \& Lê Cao, K.-A. mixOmics: an R package for 'omics feature selection and multiple data integration. PLOS Comput. Biol. 13, e1005752. https://doi.org/10.1371/journal.pcbi.1005752 (2017).

22. Ahmed, F. E. Artificial neural networks for diagnosis and survival prediction in colon cancer. Mol. Cancer 4, 29 (2005).

23. Anagnostou, T., Remzi, M., Lykourinas, M. \& Djavan, B. Artificial neural networks for decision-making in urologic oncology. Eur. Urol. 43, 596-603 (2003).

24. Jerez, J. et al. Improvement of breast cancer relapse prediction in high risk intervals using artificial neural networks. Breast Cancer Res. Treat. 94, 265-272 (2005).

25. Suzuki, K., Li, F., Sone, S. \& Doi, K. Computer-aided diagnostic scheme for distinction between benign and malignant nodules in thoracic low-dose CT by use of massive training artificial neural network. IEEE Trans. Med. Imaging 24, 1138-1150 (2005).

26. Baxt, W. G., Shofer, F. S., Sites, F. D. \& Hollander, J. E. A neural computational aid to the diagnosis of acute myocardial infarction. Ann. Emerg. Med. 39, 366-373 (2002).

27. Zini, G. Artificial intelligence in hematology. Hematology 10, 393-400 (2005).

28. Bent, P. et al. Early and intensive continuous hemofiltration for severe renal failure after cardiac surgery. Ann. Thorac. Surg. 71, 832-837 (2001).

29. Huang, L., Yu, P., Ju, F. \& Cheng, J. Prediction of response to incision using the mutual information of electroencephalograms during anaesthesia. Med. Eng. Phys. 25, 321-327 (2003).

30. Choi, J., Choi, J. \& Jung, H.-T. Applying machine-learning techniques to build self-reported depression prediction models. CIN Comput. Inform. Nurs. 36, 317-321. https://doi.org/10.1097/CIN.0000000000000463 (2018).

31. Gao, S., Calhoun, V. D. \& Sui, J. Machine learning in major depression: from classification to treatment outcome prediction. CNS Neurosci. Ther. 24, 1037-1052. https://doi.org/10.1111/cns.13048 (2018).

32. Graham, S. et al. Artificial intelligence for mental health and mental illnesses: an overview. Curr. Psychiatry Rep. 21, 1-18. https:// doi.org/10.1007/s11920-019-1094-0 (2019).

33. Helbich, M., Hagenauer, J. \& Roberts, H. Relative importance of perceived physical and social neighborhood characteristics for depression: a machine learning approach. Soc. Psychiatry Psychiatr. Epidemiol. https://doi.org/10.1007/s00127-019-01808-5 (2019).

34. Wang, S., Pathak, J. \& Zhang, Y. Using electronic health records and machine learning to predict postpartum depression. Stud. Health Technol. Inform. 264, 888-892. https://doi.org/10.3233/SHTI190351 (2019).

35. Tai, A. M. Y. et al. Machine learning and big data: implications for disease modeling and therapeutic discovery in psychiatry. Artif. Intell. Med. 99, 101704. https://doi.org/10.1016/j.artmed.2019.101704 (2019).

36. Tortajada, S. et al. Prediction of postpartum depression using multilayer perceptrons and pruning. Methods Inf. Med. 48, 291-298. https://doi.org/10.3414/ME0562 (2009).

37. Jiménez-Serrano, S., Tortajada, S. \& García-Gómez, J. M. A mobile health application to predict postpartum depression based on machine learning. Telemed. e-Health 21, 567-574. https://doi.org/10.1089/tmj.2014.0113 (2015).

38. Zhang, W., Liu, H., Silenzio, V. M. B., Qiu, P. \& Gong, W. Machine learning models for the prediction of postpartum depression: application and comparison based on a cohort study. JMIR Med. Inform. 8, e15516. https://doi.org/10.2196/15516 (2020).

39. Zhang, Y., Wang, S., Hermann, A., Joly, R. \& Pathak, J. Development and validation of a machine learning algorithm for predicting the risk of postpartum depression among pregnant women. J. Affect. Disord. 279, 1-8. https://doi.org/10.1016/j.jad.2020.09.113 (2021).

40. Luca, D. L., Garlow, N., Staatz, C., Margiotta, C. \& Zivin, K. Societal Costs of Untreated Perinatal Mood and Anxiety Disorders in the United States (Mathematica Policy Research, 2019).

41. Levis, B., Negeri, Z., Sun, Y., Benedetti, A. \& Thombs, B. D. Accuracy of the Edinburgh Postnatal Depression Scale (EPDS) for screening to detect major depression among pregnant and postpartum women: systematic review and meta-analysis of individual participant data. BMJ 371, m4022. https://doi.org/10.1136/bmj.m4022 (2020).

42. Siu, A. L. \& Force, A. T. U. P. S. T. Screening for depression in adults: US Preventive Services Task Force recommendation statement. JAMA 315, 380-387. https://doi.org/10.1001/jama.2015.18392 (2016).

43. National Collaborating Centre for Mental Health (UK). Antenatal and Postnatal Mental Health: Clinical Management and Service Guidance: Updated Edition (NICE Clinical Guidelines, No. 192. 5, CASE IDENTIFICATION AND ASSESSMENT, British Psychological Society, 2014).

44. Austin, M. P., Highet, N. \& Group, E. W. Mental Health Care in the Perinatal Period: Australian Clinical Practice Guideline (Centre of Perinatal Excellence, 2017).

45. Axfors, C. et al. Cohort profile: the Biology, Affect, Stress, Imaging and Cognition (BASIC) study on perinatal depression in a population-based Swedish cohort. BMJ Open 9, e031514. https://doi.org/10.1136/bmjopen-2019-031514 (2019).

46. Spielberger, C. D. State-trait anxiety inventory. In The Corsini Encyclopedia of Psychology, 1. https://doi.org/10.1002/9780470479 216.corpsy0943 (2010)

47. Greenwald, R. \& Rubin, A. Assessment of posttraumatic symptoms in children: development and preliminary validation of parent and child scales. Res. Soc. Work Pract. 9, 61-75. https://doi.org/10.1177/104973159900900105 (1999).

48. Feeney, J., Noller, P. \& Hanrahan, M. Assessing adult attachment. In Attachment in Adults: Clinical and Developmental Perspectives (eds Sperling, M. B. \& Berman, W. H.) 128-151 (The Guilford Press, New York, 1994).

49. Aiena, B. J., Baczwaski, B. J., Schulenberg, S. E. \& Buchanan, E. M. Measuring resilience with the RS-14: a tale of two samples. J. Pers. Assess. 97, 291-300 (2015).

50. Wagnild, G. M. \& Young, H. M. Development and psychometric evaluation of the resilience scale. J. Nurs. Meas. 1, 165-178 (1993).

51. Antonovsky, A. The structure and properties of the sense of coherence scale. Soc. Sci. Med. 36, 725-733. https://doi.org/10.1016/ 0277-9536(93)90033-Z (1993).

52. Boyce, P., Hickey, A., Gilchrist, J. \& Talley, N. J. The development of a brief personality scale to measure vulnerability to postnatal depression. Arch. Womens Ment. Health 3, 147-153. https://doi.org/10.1007/s007370170012 (2001).

53. Gelabert, E. et al. The vulnerable personality style questionnaire: psychometric properties in Spanish postpartum women. Arch. Womens Ment. Health 14, 115-124 (2011).

54. Gustavsson, J. P. et al. Swedish universities Scales of Personality (SSP): construction, internal consistency and normative data. Acta Psychiatr. Scand. 102, 217-225. https://doi.org/10.1034/j.1600-0447.2000.102003217.x (2000).

55. Wickberg, B. \& Hwang, C. P. The Edinburgh Postnatal Depression Scale: validation on a Swedish community sample. Acta Psychiatr. Scand. 94, 181-184. https://doi.org/10.1111/j.1600-0447.1996.tb09845.x (1996).

56. Vilska, S. et al. Mental health of mothers and fathers of twins conceived via assisted reproduction treatment: a 1-year prospective study. Hum. Reprod. 24, 367-377. https://doi.org/10.1093/humrep/den427 (2009).

57. Wenze, S. J., Battle, C. L. \& Tezanos, K. M. Raising multiples: mental health of mothers and fathers in early parenthood. Arch. Womens Ment. Health 18, 163-176. https://doi.org/10.1007/s00737-014-0484-x (2015).

58. Azur, M. J., Stuart, E. A., Frangakis, C. \& Leaf, P. J. Multiple imputation by chained equations: what is it and how does it work?: Multiple imputation by chained equations. Int. J. Methods Psychiatr. Res. 20, 40-49. https://doi.org/10.1002/mpr.329 (2011). 
59. Beretta, L. \& Santaniello, A. Nearest neighbor imputation algorithms: a critical evaluation. BMC Med. Inform. Decis. Mak. 16(Suppl 3), 74. https://doi.org/10.1186/s12911-016-0318-z (2016).

60. van der Laan, M. J., Polley, E. C. \& Hubbard, A. E. Super learner. Stat. Appl. Genet. Mol. Biol. https://doi.org/10.2202/1544-6115. 1309 (2007).

61. Hastie, T., Tibshirani, R. \& Friedman, J. H. The Elements of Statistical Learning: Data Mining, Inference, and Prediction 2nd edn. (Springer, 2009).

\section{Acknowledgements}

The authors would like to acknowledge Anastasia Kollia, Hanna Henriksson and Emma Bränn for valuable insights and help with data collection and management in the BASIC study. Marina Krylova, Nils Kroemer, and Hamidreza Jamalabadi for valuable insights and assistance in the initial phase of the analyses planning. Dr. Narayanan Chatapuram Krishnan for his excellent machine learning course. Subhranil Bagchi for time spent aiding in coding and students at IIT Ropar for their insights and help with coding and theoretical discussions. Prof. Inger Sundström Poromaa, Ass. Prof. Fotios Papadopoulos and all colleagues working in the BASIC research group for their contribution with critical comments and discussions. Finally, the authors would like to sincerely thank Dr. Diem Nguyen for language editing and comments.

\section{Author contributions}

A.S. conceived and designed the study. The analysis plan was decided on with the contribution of all authors. Analyses were performed by D.R.B., S.A., and S.I.I. S.A., D.R.B., and S.I.I. prepared the figures and tables. All authors made substantial contributions to the interpretation of results. S.A. wrote the first draft and all authors critically revised the manuscript and approved the final version.

\section{Funding}

Open access funding provided by Uppsala University. This study has been supported by the municipality of Uppsala and Akademiska University Hospital in Sweden, the Swedish Research foundation (523-2014-2342 and 523-2014-07605), Marianne and Marcus Wallenberg foundation and the Swedish Medical Association.

\section{Competing interests}

The authors declare no competing interests.

\section{Additional information}

Supplementary Information The online version contains supplementary material available at https://doi.org/ 10.1038/s41598-021-86368-y.

Correspondence and requests for materials should be addressed to A.S.

Reprints and permissions information is available at www.nature.com/reprints.

Publisher's note Springer Nature remains neutral with regard to jurisdictional claims in published maps and institutional affiliations.

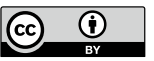

Open Access This article is licensed under a Creative Commons Attribution 4.0 International License, which permits use, sharing, adaptation, distribution and reproduction in any medium or format, as long as you give appropriate credit to the original author(s) and the source, provide a link to the Creative Commons licence, and indicate if changes were made. The images or other third party material in this article are included in the article's Creative Commons licence, unless indicated otherwise in a credit line to the material. If material is not included in the article's Creative Commons licence and your intended use is not permitted by statutory regulation or exceeds the permitted use, you will need to obtain permission directly from the copyright holder. To view a copy of this licence, visit http://creativecommons.org/licenses/by/4.0/.

(C) The Author(s) 2021 